\title{
A Coordination Scheme for Distributed Model Predictive Control: Integration of Flexible DERs
}

Costanzo, Giuseppe Tommaso; Gehrke, Oliver; Bondy, Daniel Esteban Morales; Sossan, Fabrizio; Bindner, Henrik W.; Parvizi, Jacopo; Madsen, Henrik

\section{Published in:}

Proceedings of IEEE Innovative Smart Grid Technologies Europe 2013

Link to article, DOI:

10.1109/ISGTEurope.2013.6695474

Publication date:

2013

Link back to DTU Orbit

Citation $(A P A)$ :

Costanzo, G. T., Gehrke, O., Bondy, D. E. M., Sossan, F., Bindner, H. W., Parvizi, J., \& Madsen, H. (2013). A Coordination Scheme for Distributed Model Predictive Control: Integration of Flexible DERs. In Proceedings of IEEE Innovative Smart Grid Technologies Europe 2013 IEEE.

https://doi.org/10.1109/ISGTEurope.2013.6695474

\section{General rights}

Copyright and moral rights for the publications made accessible in the public portal are retained by the authors and/or other copyright owners and it is a condition of accessing publications that users recognise and abide by the legal requirements associated with these rights.

- Users may download and print one copy of any publication from the public portal for the purpose of private study or research.

- You may not further distribute the material or use it for any profit-making activity or commercial gain

- You may freely distribute the URL identifying the publication in the public portal 


\section{A Coordination Scheme for Distributed Model Predictive Control:}

\section{Integration of Flexible DERs}

\author{
Giuseppe Tommaso Costanzo, \\ Oliver Gehrke, Daniel Esteban Morales Bondy, \\ Fabrizio Sossan and Henrik Bindner \\ Center for Electric Power and Energy \\ Technical University of Denmark, Risø Campus \\ 4000 Roskilde (DK) \\ \{guco, olge, bondy, faso, hwbi\}@elektro.dtu.dk
}

\author{
Jacopo Parvizi and Henrik Madsen \\ DTU Compute \\ Technical University of Denmark \\ 2800 Lyngby (DK) \\ \{jacop, hmad\}@dtu.dk
}

\begin{abstract}
This paper presents a control scheme based on distributed model predictive control (DMPC) for coordinating flexible distributed energy resources (DER) of heterogeneous type in the Smart Grid with minimum system integration effort. This approach can be used for reducing the peak power exchange between the grid and a cluster of units in the same feeder in pricedriven demand response applications. Preliminary simulations prove that the proposed coordination scheme for DMPC succeeds in coordinating flexible DER unit, achieving significant peak shaving when required. The rationale of this approach consists in coordinating independent units equipped with local MPC controller via simple information passing and hiding in the local controllers the units' dynamics.
\end{abstract}

Keywords-Model Predictive Control, Smart Buildings, Smart Grid, demand response, DER integration, distributed control.

\section{INTRODUCTION}

In the last two decades, factors such as increased global energy demand, speculation on fossil fuels and global warming have generated a high interest in renewable energy sources. The increase in electricity production from distributed renewable energy resources (RES), creates the need for new ways of providing ancillary services. This is important in order to maintain safe and reliable operation of the power system, both at transmission and distribution levels. Electricity production stemming from wind and solar energy can help to significantly reduce the carbon footprint of human activities and, due to favorable legislation, the installation of environmentally friendly production units in low voltage (LV) feeders has increased in the past decade. However, if corrective actions are not taken, an increased share of distributed production from renewable resources can threaten the grid stability. Therefore the integration of DERs in the distribution grid has to be operated judiciously.

It is foreseen in the near future an increase of distributed storage, provided by electric vehicles (EVs), and flexible consumption enabled by demand-side management (DSM) in buildings. This technology leads to higher flexibility and predictability of energy demand, allowing such services as peak shaving, valley filling, load shifting and real-time grid congestion management in the Smart Grid. For an insight on DSM practice, we refer to [1], [2], [3] and the references therein.
This paper presents a coordination scheme for a control architecture based on DMPC for the aggregation and coordination of multiple DER units that are connected behind a single Point of Common Coupling (PCC) in the LV grid. These units could be EVs, HVAC systems, electric water heaters and refrigerators, whose consumption is intrinsically flexible. The coordinated operation of these units allows the provision of aggregated services to the Distribution System Operator (DSO) such as peak shaving or voltage support [4], [5]. The presented approach may allow for the connection of several units with a potential high peak consumption on the same feeder while ensuring that existing flow constraints for lines, circuit breakers or transformers are respected. The main motivations for a distributed solution are computational scalability, modularity, and easy system integration.

This paper is organized as follows: Section II presents the case study and Section III the DERs models, whereas the proposed architecture for DMPC is presented in Section IV. Preliminary simulation results are presented in Section V.

\section{CASE STUdY}

This case study presents the coordination of multiple flexible DERs that may be installed in the same distribution feeder. The goal of coordinating units is to constrain the aggregated consumption/production of a cluster (group of units) to a fixed value or to a specific schedule provided by a higher-level entity in the Smart Grid, such as an aggregator or the DSO. Such service is called PowerMax and it is presented in [4]. Although the case study presented in this work considers three types of unit: building space heating and air conditioning (climate control), photovoltaic array (PV) and electric energy storage (EVs and local batteries), this coordination scheme is also suitable for other types of unit and allows units to join and leave the cluster dynamically. Figure 1 shows the configuration for this case study.

Each unit is equipped with a local model predictive controller (MPC), which optimizes the unit operation with respect to a local objective and it complies with local and global constraints. Controllers' local objective can range from minimizing operating costs to maximizing comfort, while local constraints ensure minimal comfort requirements and safe unit operation. The units in the cluster have also to respect a global constraint which relates to the power flow at the point of 


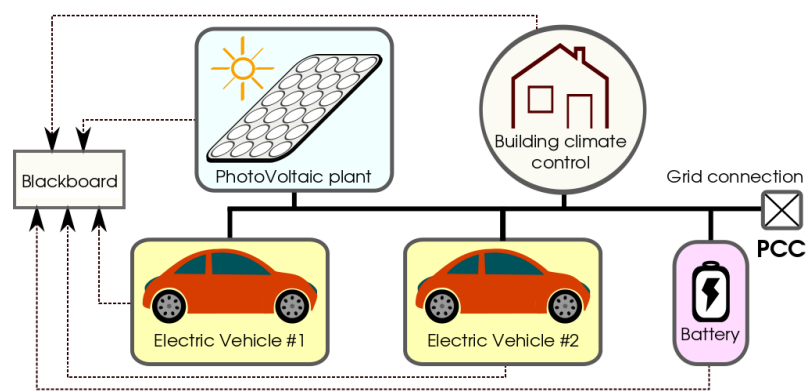

Fig. 1. Case study setup.

connection between the cluster and the main grid. Each unit, by retrieving information about other units consumption and the PCC power limit from the cluster blackboard, computes the local power plan by solving a constrained optimization problem. The next section presents, for each unit type, the model and the operational requirements (local constraints).

\section{MODELS OF DERS}

1) Building and space heating: the model for building heating demand is taken from [6] (model $\left.T_{i} T_{e} T_{h}\right)$, where a single room-equivalent model is used for an office building of eight rooms equipped with electric space heating and air conditioning units. Model 1, which has three states, represents the dynamics of the (equivalent) room internal temperature, the (equivalent) heater temperature and the envelope (building insulation) temperature:

$d T_{i}=\frac{1}{C_{i}}\left[\frac{1}{R_{i e}}\left(T_{e}-T_{i}\right) d t+\frac{1}{R_{i h}}\left(T_{h c}-T_{i}\right) d t+A_{w} \Phi_{s} d t\right]+\sigma_{i} d w_{i}$

$d T_{e}=\frac{1}{C_{e}}\left[\frac{1}{R_{i e}}\left(T_{i}-T_{e}\right) d t+\frac{1}{R_{e a}}\left(T_{a}-T_{e}\right) d t\right]+\sigma_{e} d w_{e}$

$d T_{h c}=\frac{1}{C_{h}}\left[\frac{1}{R_{i h}}\left(T_{i}-T_{h c}\right) d t+\Phi_{i n} d t\right]+\sigma_{h} d w_{h}$

where $\Phi_{i n}$ and $\Phi_{s}$ are respectively the electric power consumed for climate control and the solar irradiation. Although Model 1 does not include the air conditioning units, in this study, as a simplifying assumption, the air conditioner dynamics is assumed equivalent to the heater dynamics. The fact that the heater dynamics is about two orders of magnitude faster than the room dynamics justifies the choice of assuming the AC unit "fast enough", as the heater is, with respect to the room heat dynamics. Therefore the cooling effect of air conditioning is accounted with a negative sign on $\Phi_{i n}$ and a multiplicative factor of 3 (average COP from AC units datasheet). Finally $T_{i}, T_{e}$ and $T_{h c}$ are, respectively, the room internal temperature, the envelope temperature and the heating/cooling module temperature.

2) Batteries and EVs: in this work the EVs are modeled as batteries and driving patterns are considered and simulated as disturbances to the MPC. A detailed model of electric storage units is presented in [7], from which it emerges that, for lifetime purposes, the batteries should be operated between $20 \%$ and $90 \%$ of the state of charge (SOC). Moreover, when operating in this band, the batteries can be charged and discharged at constant current and can be reasonably modeled as integrators of rated efficiency. The support batteries differ from the EVs by capacity and rated power, therefore a generic state space model for this type of units is presented below:

$$
\begin{aligned}
& d x=A_{s} x d t+B_{s} u d t \\
& S O C=C_{s} x
\end{aligned}
$$

where $u$ is the power flow at the inverter in $k W, x$ is the battery state of charge in $k W h$ and $S O C$ is the battery state of charge normalized between 0 and 1 and it is related to the battery rated capacity.

3) $P V$ array: this unit is supposed to operate at maximum power point (MPP) and the unit local controller, using the forecast of solar radiance, wind speed and external temperature, publishes in the blackboard the expected power production using the model presented in [8], [9]. Every time the weather forecast is updated the PV controller accordingly updates the PV production plan in the blackboard. In this study, as simplification, the PV unit publishes a production plan which consists in the actual produced power from a real PV plant with added a normally distributed noise of $10 \%$ of the power measured at each instant. Then, at simulation time, the effective power produced by the unit is given by the real measurements decurted of the noise. This approach is considered in order to simulate inaccuracies on PV modeling and weather forecasts.

\section{CONTRol ARChitecture}

The computational effort for solving MPC problems generally grows at a superlinear rate with the number of state variables involved. The exact order is problem specific and depends on the coupling between the state variables as well as the chosen solving method. Managing multiple DERs with a single, feeder-wide MPC controller would not only require a large amount of DER state information to be shared with the central controller. The computational effort of such a setup would also scale badly for larger numbers of units. An established approach for solving the curse of dimensionality relies on the decomposition of the MPC problem into smaller subproblems which can be solved independently and locally. Convergence towards the overall goal is then achieved through a coordination mechanism, i.e. by communication between the individual solvers. An overview and categorization of distributed MPC variants is given in [10], [11]. In the presented approach, the MPC computations are carried out at unit level by the local unit controller. In order to meet the system constraint of limited power flow at the PCC, controllers have to coordinate because the fulfillment of the system constraint may not be derived only by measuring physical variables observable by the individual units.

Coordination between autonomous entities in a distributed system has been an active area of research for many years [12]. Many different ways of achieving such coordination have been proposed; however, as with many engineering problems, no one-size-fits-all solution exists and the specific trade-offs of a particular method have to be weighed against the needs of the distributed system in question. These needs may include scalability of the solution, synchronization speed, access concurrency, fault tolerance, level of trust between entities, convergence speed and others. The communication effort of the distributed MPC system presented in this paper needs to be scalable to a certain degree, but the speed of synchronization is not critical. A common approach for this 
type of system is the use of a tuple space for information exchange, typically implemented as a blackboard [13], i.e. a central data repository whose content all processes can retrieve and to which all processes can write. For this type of solution, the communication effort - as defined by the message count - for each iteration scales linearly with the number of units. A notable drawback is that a single point of failure is introduced at the blackboard itself which could be partially mitigated by adding a replication and failover mechanism for the blackboard.

\section{A. Algorithm for MPCs coordination}

In the presented scheme all units access the blackboard asynchronously, retrieve the power plans of the other units, compute their own plan accordingly and publish it in the blackboard. Units have exclusive access to the blackboard, which is a resource that is blocked by one unit at the time during the processes of data retrieving, power plan computing and data publishing. As soon as the available power at the PCC decreases, units tend to reduce their consumption (or production). However, if the operating or comfort requirements of a unit cause a violation on the constraint on the PCC power flow, a minimal power plan that minimizes the exceeding power at the PCC, called power over max, is published in the blackboard and the consensus flag related to that plan is set to false. Units keep calling the blackboard if they need to update their operation plan until the consensus flag is set to true or the iterations of such process reach a predefined limit, $L$, which is set equal to all units and serves to avoid deadlocks. When those conditions hold, the units are entitled to operate according to the local power plan for the next time frame. Thanks to such mechanism the minimal operation and comfort requirements of units are always satisfied by the local controllers, at the price of occasional violations of the limits on the PCC power flow in the case of over stringent constraint. Algorithm 1 presents the coordination scheme for DMPC, while the following subsections present the unit local MPC formulation for the building climate control and control of electric storage.

\section{B. Building controller}

The building controller uses a discretized version of Model $1(\Delta t=30 \mathrm{~min})$ and keeps the internal temperature at time $t, T_{i, t}$, within the comfort bounds $\left[T_{\min , t}, T_{\max , t}\right]$ and trades off energy expenses with user comfort by solving the following optimization problem:

$$
\begin{aligned}
& \min _{\Phi_{h, t}, \Phi_{c, t}, \vartheta_{t}} J=\sum_{t=1}^{N}\left[\left\|T_{i, t}-R_{t}\right\|_{Q}^{2}+p_{t}\left(\Phi_{h, t}+\Phi_{c, t}\right)+\rho \vartheta_{t}\right] \\
& \text { subject to : } \\
& x_{t+1}=A_{b} x_{t}+B_{b} \Phi_{h, t}+F_{b} \Phi_{c, t}+E_{b} T_{a, t}+S_{b} \Phi_{s, t} \\
& T_{i, t}=C x_{t} \\
& 0 \leq \Phi_{h, t} \leq U_{\max , t} \\
& 0 \leq \Phi_{c, t} \leq C_{\max , t} \\
& T_{\min , t} \leq T_{i, t} \leq T_{\max , t} \\
& \vartheta_{t} \geq 0 \\
& P C C_{\min , t}-\vartheta_{t} \leq \Psi_{t}+\Phi_{h, t}+\Phi_{c, t} \leq P C C_{\max , t}+\vartheta_{t}
\end{aligned}
$$

The objective function in Eq. 3a is composed by three terms. The first term penalizes the deviation of $T_{i, t}$ from the setpoint, $R_{t}$; the energy expense is minimized in the second

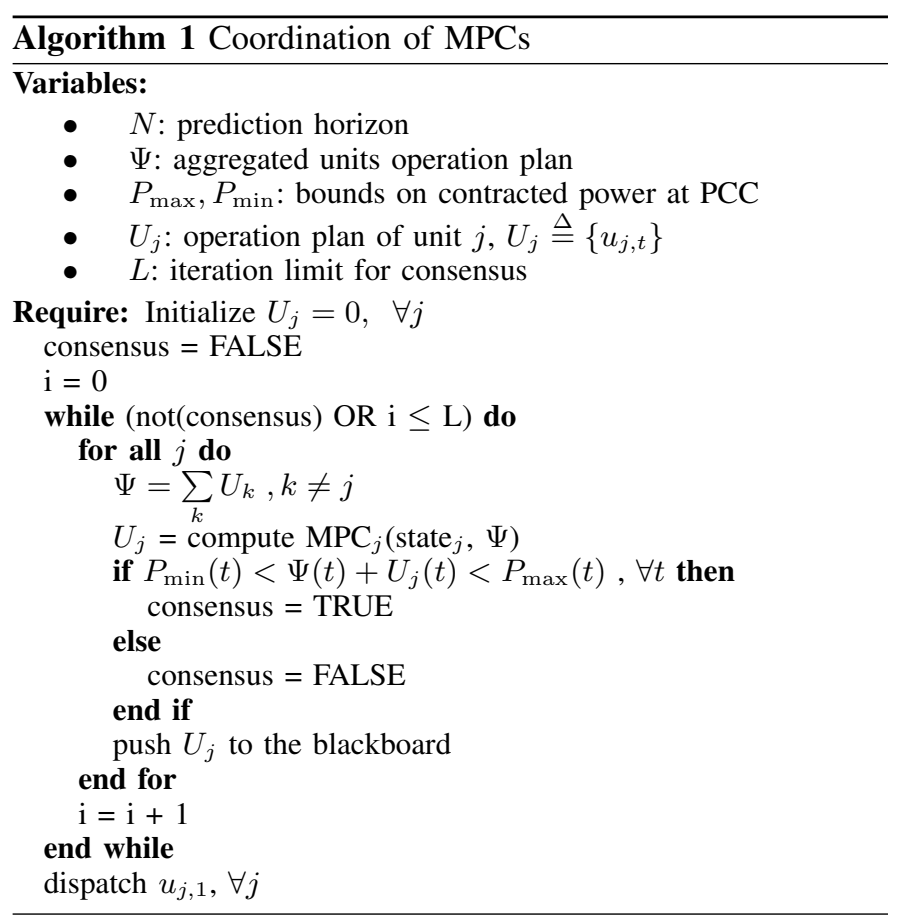

term, where $p_{t}$ is the energy price over the prediction horizon $N$ taken from the Nordpool Elspot market; finally power over $\max , \vartheta_{t}$, is minimized in the third term with the parameter $\rho$.

The MPC operates the heater and the air conditioner independently, so that the control input $\Phi_{i n}$ in Eq. 1c is divided in: $\Phi_{h, t}$ for heating and $\Phi_{c, t}$ for cooling. In this way the discretized model in Eq. $3 \mathrm{~b}$ presents two control inputs $\left(\Phi_{h, t}\right.$ and $\left.\Phi_{c, t}\right)$ and two disturbances $\left(T_{a, t}\right.$ and $\left.\Phi_{s, t}\right)$. Equations $3 b$ and $3 \mathrm{c}$ represent the building thermal dynamics for the MPC, Eq. $3 \mathrm{~d}$ and $3 \mathrm{e}$ set the bounds on control inputs, whereas the comfort bounds for the building internal temperature are stated in Eq. 3f. Finally, violations of limits on the PCC power flow are allowed by the soft constraints in Eq.3g and $3 \mathrm{~h}$. The optimization problem defined in Eq. 3 is reformulated as a least squares problem, which is solved via quadratic programming algorithm as presented in [14].

\section{Battery and EV controller}

The controller for the electric storage strives at minimizing the energy cost while keeping the SOC within given bounds. The MPC problem is formulated as follows:

$$
\begin{aligned}
& \min J=\sum_{t=1}^{N}\left[p_{t} u_{t}+\rho \vartheta_{t}\right] \\
& \text { subject to }: \\
& x_{t+1}=A_{s} x_{t}+B_{s} u_{t} \\
& S O C_{t}=C_{s} x_{t} \\
& U_{\min , t} \leq u_{t} \leq U_{\max , t} \\
& S O C_{\min , t} \leq S O C_{t} \leq S O C_{\max , t} \\
& P C C_{\min , t}-\vartheta_{t} \leq \Psi_{t}+u_{t} \leq P C C_{\max , t}+\vartheta_{t}
\end{aligned}
$$

The cost function is expressed in Eq. 4a, which consists in a term for minimizing the energy cost $\left(p_{t}\right.$ is the Nordpool Elspot price) and a term, $\rho$, for minimizing the power over max. Constraints in Eq. $4 \mathrm{~b}, 4 \mathrm{c}$ implement the energy 
storage dynamics, while Eq. $4 \mathrm{~d}$ accounts for inverter power capabilities. Constraints in Eq. 4e allow the MPC controller to keep the battery state of charge between given operational bounds. This constraint can be used to bring the SOC to a convenient level, accounting for the users driving patterns and the unit's availability. If the controlled unit is a battery, the SOC is not time dependent. Finally, Eq. $4 \mathrm{f}$ penalizes the excess of power flow at the PCC. A similar MPC formulation for vehicle charging is presented in [15].

In the control scheme proposed in this work no computation is carried out at the data repository level, which can be as simple as an html page. In this way the devices optimization is entirely distributed to a local level. Using a blackboard with asynchronous units access and negotiation allows to keep both system and information exchange simple; moreover there is no need for hierarchical coordination, since this is achieved by means of coupling constraints in the local MPCs. All of these factors enhance the system interoperability, allowing to integrate and coordinate devices of heterogeneous type and with totally different operation requirements and constraints. Ultimately, if a data repository is not available, each controller can broadcast its plan to the other controllers. In this case the number of messages exchanged considerably grows with the number of units per group.

\section{Simulation Study}

The simulation study shows the effectiveness of the proposed algorithm in limiting the aggregated power flow of a cluster of units composed by 100 buildings, 10 local batteries, 20 large EVs, 20 small EVs and 20 PV modules. This population of units is created by using the models from literature with parameters that are normally distributed with a $10 \%$ variance around the nominal value. Figure 2 presents the aggregated power flow at the PCC without units coordination (black line, with peaks up to $+420 \mathrm{~kW}$ and $-320 \mathrm{~kW}$ ) and with units coordination (green line). It is shown that the proposed algorithm successfully limits the aggregated power flow between the given bounds of $\pm 200 \mathrm{~kW}$.

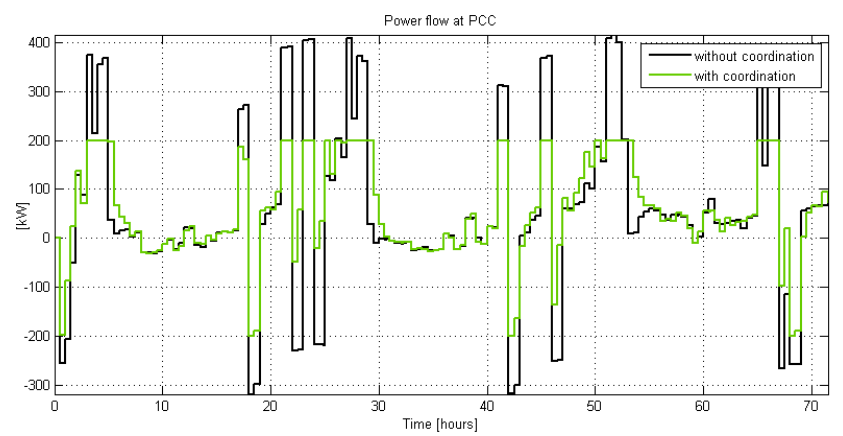

Fig. 2. Power flow at the PCC: units coordination v.s. units non-coordination.

Note that the coordination scheme has no effect when the units' aggregated consumption is spontaneously below the given limit of $\pm 200 k W$, eg. the green and black line are superposed in the time frame between $30 \mathrm{~h}$ and $40 \mathrm{~h}$ (Fig. 2). Figure 3 shows the power exceeding the PCC limit versus the number of coordination iterations, for different maximum power requirements. It emerges that the algorithm converges in two iterations if the available power at the PCC is sufficient

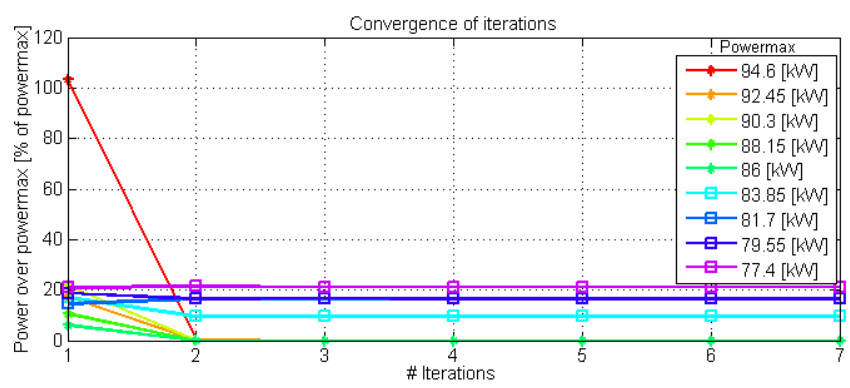

Fig. 3. study of algorithm convergence: exceeding power flow at the PCC v.s. number of iterations.

to satisfy the units minimum requirements. Conversely, if the available power is not sufficient, the units controllers keep trying to coordinate without success and the consensus is not reached. Such limit for the given setup is between 86 and 84 kilowatts. Focusing now on single units' operation, Fig. 4 and Fig. 5 show the range of all buildings' internal temperature and power consumption, respectively for the cases of units noncoordination and units coordination. By inspecting Fig. 4 and

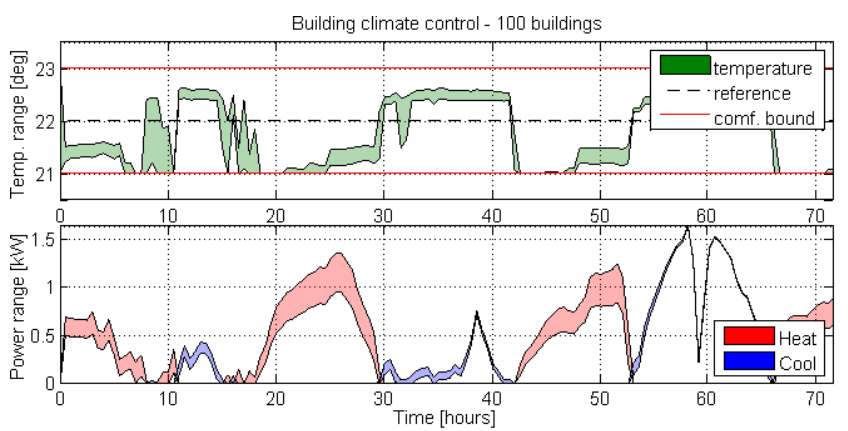

Fig. 4. Building thermal management without units coordination.

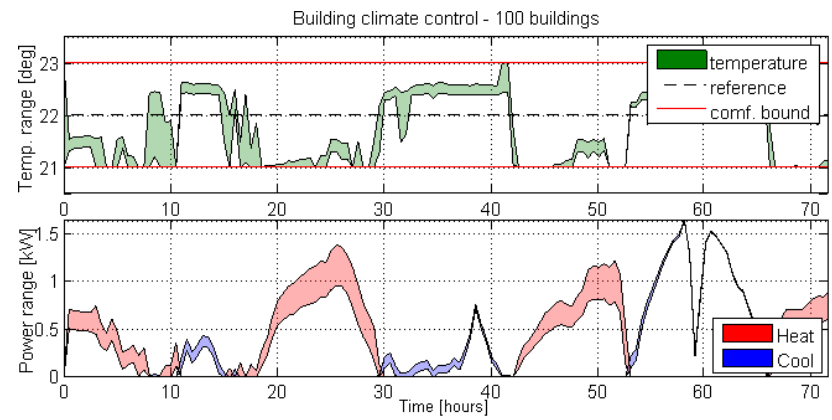

Fig. 5. Building thermal management with units coordination.

Fig. 5 it emerges that the buildings contribute in few occasions to shape the cluster consumption.

Figures 6 and 7 show the operation of the energy storages: batteries, small and big EVs. The range of units' SOC and power flow are shown for the cases of units non-coordination and coordination. It is observed in Fig. 6 that, when units are not coordinated, the control inputs lay in a narrow range. This behavior is due to the economic formulation of the MPCs: all units tend to charge when the price of energy is low and discharge when the price is high, causing peaks in the power flow at the PCC. When units are coordinated via DMPC, the trajectories of control inputs and SOC stay within a wider range, showing the units flexible operation (Fig. 7). 

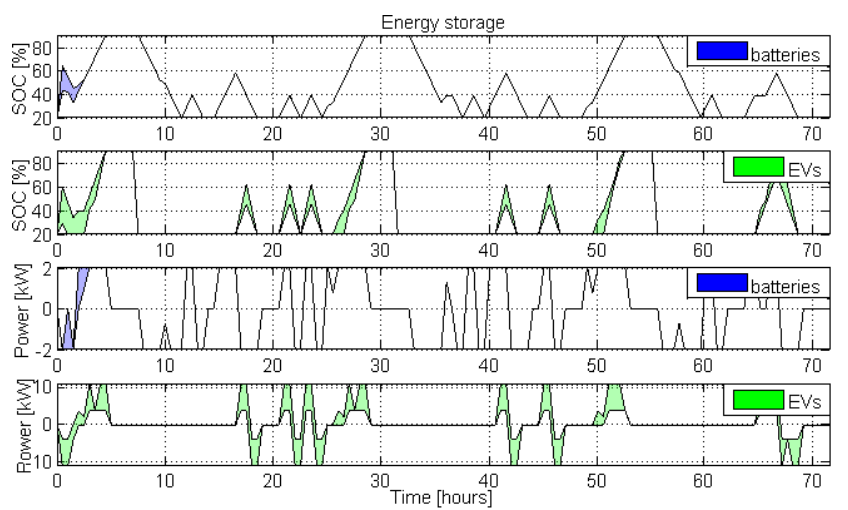

Fig. 6. Battery operation without units coordination: range of units' SOC and power flow.
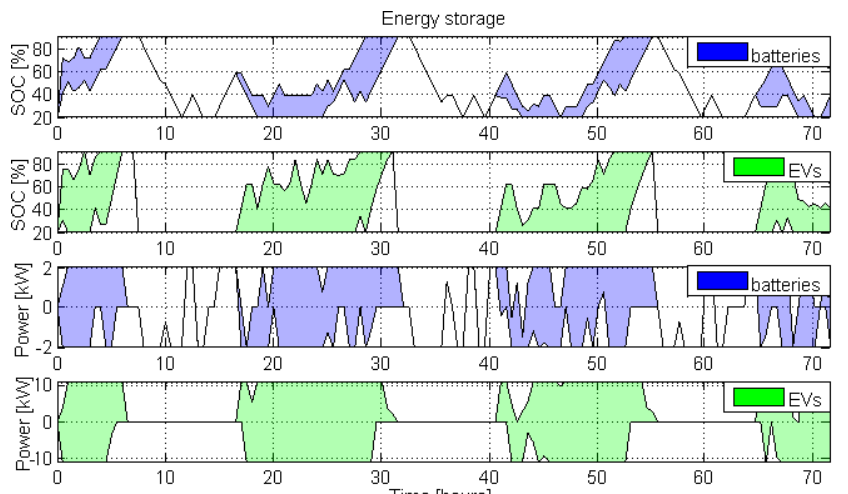

Fig. 7. Battery operation with units coordination: range of units' SOC and power flow.

Fig. 8 shows the predicted and realized PV production, and the NordPool Elspot price.
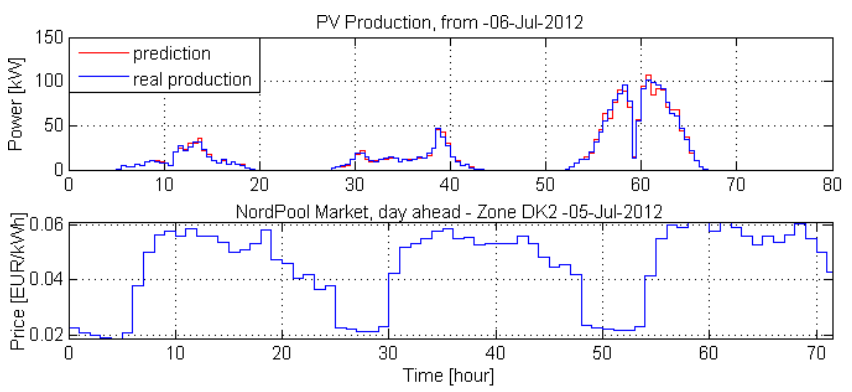

Fig. 8. Energy price and PV production.

The simulation study shows that the proposed architecture is capable of coordinating units in a cluster so as to shave the peak demand. However, although the algorithm convergence and optimality of the solution have not been formally proven, simulation trials show that given sufficient available power at the PCC to serve the units minimum requirements, the coordination scheme is successful and units flexibility is fully exploited. Moreover, it has been observed in simulation with smaller clusters that the speed of convergence does not depend by the cluster size.

\section{CONCLUSiONS}

This paper presents an approach for the integration of flexible DERs in the Smart Grid based on DMPC coordination via coupling constraints. In the presented study, this control scheme allows minimizing the impact of PV plants and EVs in the distribution grid and the advantages brought from the blackboard-based coordination include scalability and easy system integration. It is planned as future study to investigate fairness of resource allocation among units, assess reliability problems in case of downtime of the blackboard and comparison with other DMPC algorithms.

\section{ACKNOWLEDGMENTS}

The authors acknowledge the financial support of iPower, a project within the Danish Strategic Platform for Innovation and Research within Intelligent Electricity (www.ipower-net.dk).

\section{REFERENCES}

[1] C. W. Gellings, "The concept of demand-side management for electric utilities," Proceedings of the IEEE, vol. 73, no. 10, pp. 1468-1470, 1985.

[2] L. Chan, N. Li, S. H. Low, and J. Doyle, "Two market models for demand response in power networks," in IEEE SmartGridComm 2010, Gaithersburg, MD, USA, October 2010, pp. 4569-4574.

[3] Z. Yua, F. Haghighata, B. C. Fungb, and H. Yoshinoc, "A decision tree method for building energy demand modeling," Energy \& Buildings, vol. 42, no. 10, pp. 1637-1646, 2010.

[4] N. C. Nordentoft, Y. Ding, L. H. Hansen, P. D. Cajar, P. Brath, H. W. Bindner, and C. Zhang, "Development of a dso-market on flexibility services," iPower, Tech. Rep., 2013.

[5] O. Gehrke and F. Isleifsson, "An aggregation friendly information model for demand side resources," in Local Computer Networks (LCN), 2010 IEEE 35th Conference on, 2010, pp. 1019-1023.

[6] P. Bacher and H. Madsen, "Identifying suitable models for the heat dynamics of buildings," Energy and Buildings, vol. 43, no. 7, pp. 1511 - 1522, 2011.

[7] F. Marra, G. Y. Yang, C. Traholt, E. Larsen, C. Rasmussen, and S. You, "Demand profile study of battery electric vehicle under different charging options," in Power and Energy Society General Meeting, 2012 IEEE, 2012, pp. 1-7.

[8] W. De Soto, S. Klein, and W. Beckman, "Improvement and validation of a model for photovoltaic array performance," SOLAR ENERGY, vol. 80, no. 1, pp. 78-88, 2006.

[9] C. Koch-Ciobotaru, L. Mihet-Popa, F. Isleifsson, and H. Bindner, "Simulation model developed for a small-scale pv-system in a distribution network," in Proceedings of the 8th International Symposium on Applied Computational Intelligence and Informatics-SACI 2012, 2012, pp. 257261.

[10] R. Negenborn, B. D. Schutter, and J. Hellendoorn, "Multi-agent model predictive control: A survey," Delft University of Technology, Tech. Rep. 04-010, August 2009.

[11] "Architectures for distributed and hierarchical model predictive control a review," Journal of Process Control, vol. 19, no. 5, pp. 723 - 731, 2009.

[12] T. W. Malone and K. Crowston, "The interdisciplinary study of coordination," ACM Comput. Surv., vol. 26, no. 1, pp. 87-119, Mar. 1994. [Online]. Available: http://doi.acm.org/10.1145/174666.174668

[13] V. Jagannathan, R. Dodhiawala, and L. Baum, Blackboard Architectures and Applications, ser. Perspectives in Artificial Intelligence. Academic Press, 1989.

[14] R. Halvgaard, N. Poulsen, H. Madsen, and J. Jorgensen, "Economic model predictive control for building climate control in a smart grid," in Innovative Smart Grid Technologies (ISGT), 2012 IEEE PES, 2012, pp. $1-6$.

[15] R. Halvgaard, N. Poulsen, H. Madsen, J. Jorgensen, F. Marra, and D. Bondy, "Electric vehicle charge planning using economic model predictive control," in Electric Vehicle Conference (IEVC), 2012 IEEE International, 2012, pp. 1-6. 